\title{
cloudMAGNET
}

\section{A prototype for climatically active light-weight skins}

\author{
Andrew John Wit ${ }^{1}$, Rashida $\mathrm{Ng}^{2}$ \\ ${ }^{1,2}$ Temple University \\ 1,2\{andrew.wit|rashida.ng\}@temple.edu
}

This paper describes a potential for the integration of micro-encapsulated phase change material (mircoPCM) into lightweight skins as a means of regulating internal climatic conditions of volumetric objects. Viewed through the lens of the recently completed series of quarter-scale cloudMAGNET prototypes tested in the cloud forests of Monteverde, Costa Rica, this research utilized a wound, flexible carbon fiber framework and a lightweight fabric skin coated with varying densities of microPCM. The prototypes were monitored using real-time collection of climate data throughout the testing. In this paper we will demonstrate how climatic variables such as temperature, humidity, and pressure can be passively manipulated by varying the form and energy storage properties of materials without the use of active mechanical systems. Produced to bring awareness to the rising cloud levels within the Monteverde cloud forest, this research is intended to explore the fundamental relationships of material, energy and form. Beyond these objectives, the paper will also illustrate how these methods can be more broadly applied to the development of thermal-regulating lightweight tensile structures. Such innovations could be utilized as a method for the reimagining the architectural design and production processes allowing for the emergence of new typologies of environmentally self-mediating architecture.

Keywords: material performance, phase change material, carbon fiber reinforced polymers, computation

\section{INTRODUCTION}

Architecture and the construction industry have a large impact on the climate through the process of material production, as well as throughout the full lifetime of the buildings we create. With such long term environmental consequences, the discipline bears a responsibility to reimagine the materials, processes and techniques with which we con- struct buildings, as well as for designing for how they interact with their surrounding environment in both the short and long term.

Although recent advances in computation, materials science and novel methods for production, such as robotic fabrication, 3D and 4D printing, have redefined paradigms of making in many design disciplines, architecture has not yet realized the same 
level of widespread innovation [Gramazio/Kohler, Rael/San Fratello, Tibbets]. The pace of adoption of new materials and technologies within the building sector has been slower, as traditional materials remain in use despite notable inefficiencies, such as energy intensive manufacturing processes and poor thermal performance. cloudMAGNET seeks to reveal often hidden relationships between materials and the environments in which they are situated. Furthermore, it aims to provoke innovations in the field that leverage these relationships to improve the environmental and experiential performance of architecture.

Designed for the cloud forests of Monteverde, Costa Rica, the project brings attention to the rising cloud levels that are threatening the forest ecosystem, while simultaneously rethinking the role of the architectural skins (i.e. façades). Where in contemporary architecture the façade has acted as a static enclosure forming a physical separation between inside and out, this research rather investigates skin as an active mediating agent, modifying environments without the use of electromechanical systems or operable fenestrations.

\section{BACKGROUND}

cloudMAGNET was developed as a series of kite prototypes testing the potential to manipulate internal environmental conditions such as temperature and humidity through form, pressure differentials and the integration of microPCM coated lightweight skins. Initial prototypes included two quarter-scale kites (K1 and K-2) and another one-fifth scale (K-3) kite that were all tested in Monteverde, Costa Rica [Figure 01].

Each unique prototype was designed to increase the internal humidity of air as it flowed through the funnel shaped kites by reducing the pressure and lowering the temperature in order to stimulate condensation of the water vapor into a fine mist of clouds. The results of this research combining thermal mediating microPCM could be transferred to architectural skins and smart building forms that are utilized to create performative skins and shelters for environmental control. Throughout the develop- ment of the installation, several research areas were explored:

- Design of the form of the kite: The relationship of form to changes in the velocity and pressure differentials were explored through the use of computational fluid dynamics (CFD) software. The research team utilized an iterative process to design, model and analyze each of the designs. The geometry of the form was based on Bernoulli's Principle of airflow that correlates reduced air pressure with increased wind speed with the flow of air from a wider opening through a narrow throat. Furthermore, the form of the prototypes were designed to naturally rotate as wind passed through in order to maximize the surface area of the microPCM exposed to the sun. The variables in the kite design were: A) Distance between air inlet \& outlet; B) Shape of the inlet \& outlet; C) Size of the air inlet \& outlet; D) Finally the rotational angle between the inlet \& outlet. Following numerous CFD digital studies and physical wind tunnel visualizations of 3D printed models, a single design was chosen for further design refinement through the fabrication of large scale prototypes for testing (K-1, K-2, and K-3). [Ng, Wit, Hsieh, 2018]

- Design and fabrication of the CFRP frame: The design and fabrication of the CFRP structure was coordinated with the final form of the kite skin following the CFD analysis and wind tunnel visualizations. The form and structural design process required an iterative process as the CFD generated forms needed to be refined based on the fabrication process required for the tension based fiber winding process of the CFRP frame. Because the prototypes needed to not only be lightweight but also robust and flexible at the same time, $24 \mathrm{k}$ $(24,000)$ resin pre-impregnated carbon fiber reinforced polymer (CFRP) tow was utilized for the structural frame [Figure 02]. As this specific fiber required kiln based firing for cur- 
Figure 1

The three prototypes produced for testing in Monteverde

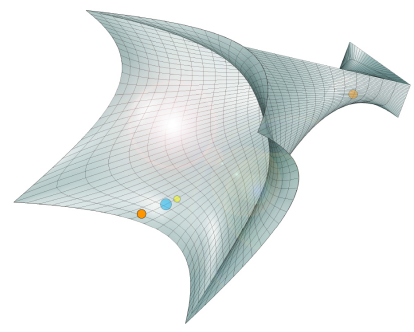

KITE 1

Size: $\quad$ Large $\left(30^{\prime \prime} \times 30^{\prime \prime} \times 48^{\prime \prime}\right)$

Design: $180^{\circ}$ Rotation

Fabric: Dyneema CT5K.18 welded w/ CTPSA tape

PCM: None

Data: (1) Feather M0 Micro-controller (2) BME280 Temp/Humidity/Pressure (1) RTC_DS3231 Real-time

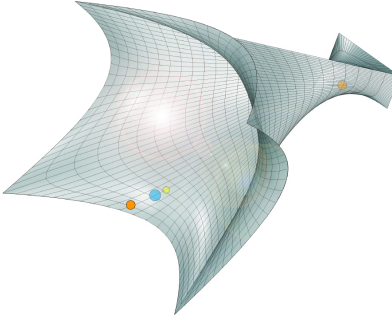

KITE 2

Large $\left(30^{\prime \prime} \times 30^{\prime \prime} \times 48^{\prime \prime}\right)$

$180^{\circ}$ Rotation

Dyneema CT5K.18 welded w/ CTPSA tape

Rubitherm Microencapsulated PX 27 $50 \%$ surface area w/ $3 \mathrm{M}$ transfer tape

(1) Feather MO Micro-controller (2) BME280 Temp/Humidity/Pressure

(1) RTC_DS3231 Real-time

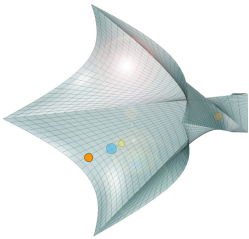

KITE 3

Small $\left(24^{\prime \prime} \times 24^{\prime \prime} \times 36^{\prime \prime}\right)$

$90^{\circ}$ Rotation

Dyneema CT5K.18 welded w/ CTPSA tape

Rubitherm Microencapsulated PX 27 $50 \%$ surface area w/ $3 \mathrm{M}$ transfer tape

(1) Feather MO Micro-controller (2) BME280 Temp/Humidity/Pressure (1) RTC_DS3231 Real-time ing, each of the prototypes structures were prefabricated in Philadelphia and shipped by air freight to Monteverde. To allow for the compact transport it was necessary for each prototype to not only nest within each other, but also have varying levels of flexibility allowing the structure to be compressed along the large inlet. To achieve this, a coreless CFRP winding method was developed utilizing a reconfigurable steel frame with a reusable tensioned cable formwork which allowed for the creation of a structure with varying levels of rigidity along its length enabling the kite to be compressed from a diameter of $1.5 \mathrm{~m}$ to $.4 \mathrm{~m}$. [Wit, 2018]

- Fabrication of the lightweight skin: To modify the internal climatic conditions of the installation, a robust lightweight skin was developed to fit within the CFRP structural envelope. This skin consisted of three elements which will be described in detail below: A) A panelized ultra-high molecular weight polyethy- lene (UHMwPE) fabric creating the form of the kite skin; B) A variable patterned coating of micro-encapsulated PCM; and C) A series of pockets to house electronics and sensors. To ensure that the forms were developable as a tensile membrane structure, each one was simulated, verified and modified utilizing the Rhino 3D plug-in MPanel. The plugin, through the input of the UHMwPE fabric characteristics allowed for the precise calculation of the final geometry of the complex doubly curved surfaces translated into flat panel surfaces.

- Programming of climatic data sensors: Finally, to record real-time climatic variations within the installation, custom microcontrollers were assembled with a tethered Arduino Nano board connected to a series of sensors that measured temperature, pressure and the humidity of the air. One sensor was located at the inlet of each kite; another was placed at the air outlet. 
As the first two elements, form and frame have been described extensively in other papers (see citations), this paper will focus on the design and fabrication of the skin and the electronic sensors. Finally, it will provide further analysis of the performative results collected and their potential applications for environmental mediation with lightweight tensile skins.

\section{LIGHTWEIGHT SKINS}

Although the form and structure of the kite were essential elements to the kites function, the production of these elements was more predictable as they were designed, developed and pre-fabricated within fully equipped facilities. The skins on the other hand, not only needed to be durable and performative, but also were required to be assembled on-site with few specialized tools. Additionally, the prototypes were designed to reduce the amount of waste produced on site.

The form of the lightweight fabric closely followed that of the CFRP structure as the skins were stretched into tension by the CFRP frame. Throughout the production of the skins, there were three important areas of exploration:

\section{Materials}

As strength, minimal weight and ease of production were all extremely important in the production of the skin of the prototypes, a laminated non-woven ultrahigh molecular weight polyethylene (UHMwPE) fabric, Dyneema CT5k.18, 50g/sqm was chosen as the skin. Previously marketed under the brand name, cuben fiber, Dyneema is a non-woven, non-stretch, dimensionally stable, and tear resistant fabric, which utilizes bidirectional UHMwPE fibers that are heat welded between two ultra thin carrier sheets. Initially developed for the sailing industry, Dyneema fabric is also waterproof and UV resistant. According to its manufacturer, Royal DSM, Dyneema is 30\% lighter than nylon/polyester, $45 \%$ lighter than aramid, and fifteen times stronger than steel by its weight to weight ratio. UHMwPE fibers are integrated into an increasing range of composite fabrics, such as carbon
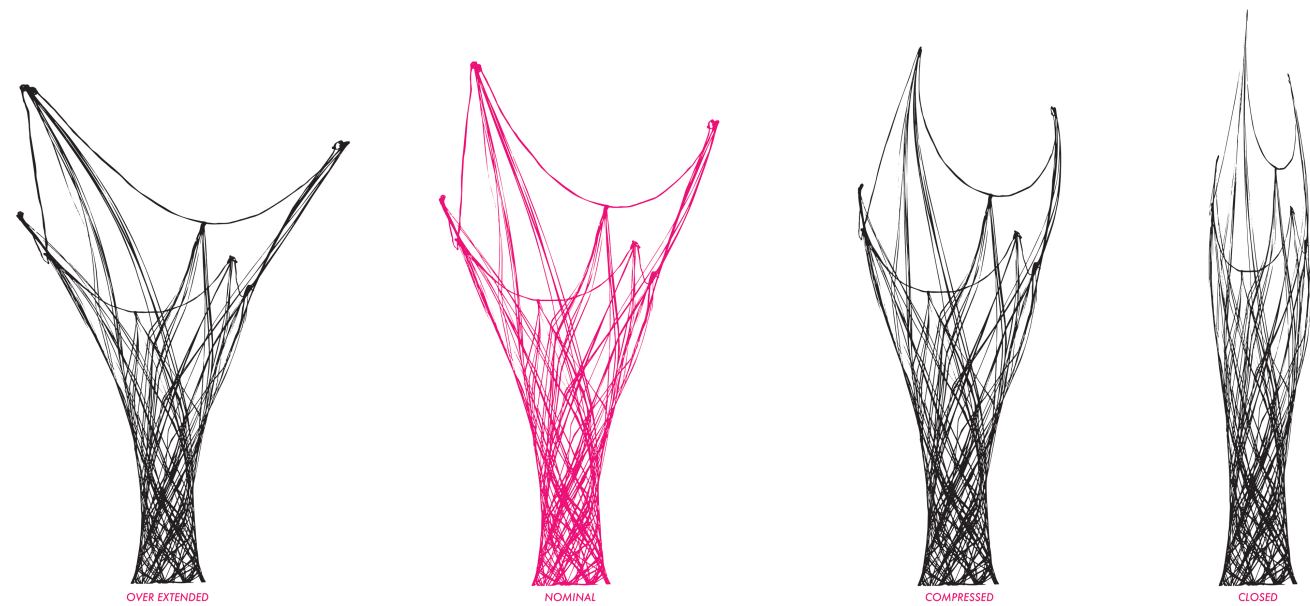

Figure 2 Flexing of the CFRP structure 
fiber, denim, knits, and wovens, in order to improve their strength and durability.

It was found during prototyping that Dyneema fabric also provided a printable surface that allowed the patterns of each panel to be transferred directly onto the surface using a wide format plotter. Additionally, as the sheets are extremely thin, they are easily workable with simple tools such as scissors or modeling knives. The connecting of sheets in the production of the overall form was also easily achievable through the use of fabric welding tape as further described below.

Figure 3 Prototype edges

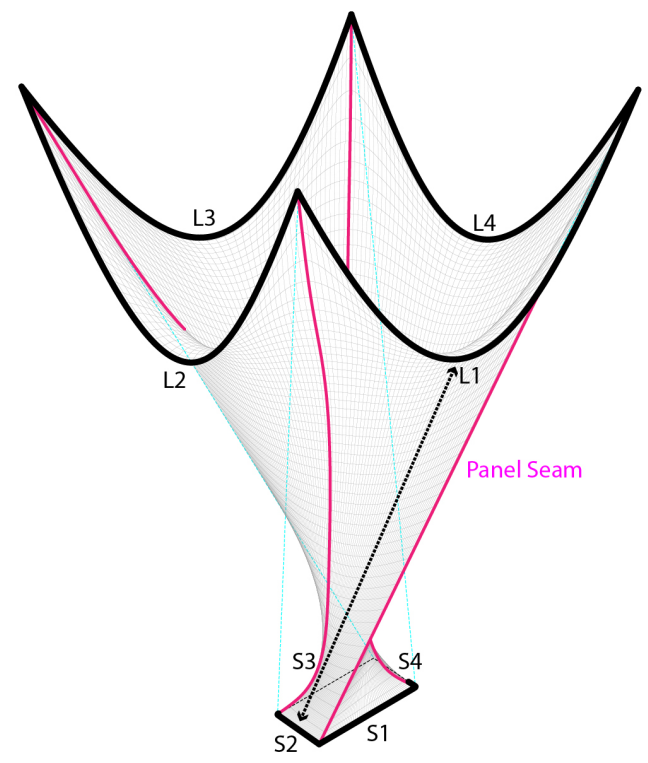

\section{Production}

Each of the three prototypical skins were produced in a similar fashion despite variations in scale between $\mathrm{K}-1 / \mathrm{K}-2$ and $\mathrm{K}-3$. Following initial CFD analysis, wind tunnel visualizations, and the development of CFRP winding techniques, the desired fabric forms were re- generated in a way that would allow them to be produced from flat stock material. To do this, each form was remodeled through the tensile fabric modeling plug-in for Rhino 3D MPanel which created a simulated relaxed skin model.

As both the inlet and outlet sizes and shapes were predefined through the design process, their edges were extracted as a means of creating a fixed edge tensile structure within the MPanel workspace. The large opening edges were labeled L1, L2, L3, L4 and small as S1, S2, S3, S4 [Figure 3].

MPanel joined edge meshes (joined edge meshes are necessary to allow for a seamless flow between relaxed panels) with twenty subdivisions in both the warp and weft direction were then created by drawing meshes between opposing edges. As the inlet and outlet were rotated 90 degrees as a means of creating rotation as air passed through the structure, the meshes were joined as follows: L1 to S2; L2 to S3; L3 to S4; and L4 to S1 creating panels P1, P2, P3 \& P4 [Figure 4]. The initial output of which creates four connected ruled meshes with straight edges between each of the joined panels. Following the creation of the mesh panels, all four panels were selected and relaxed as a single, continuous four panel joined edge mesh which creates the final relaxed tensile membrane. It was important at this point to compare the original designed form to the form generated through the tensile modeling software. If the variations between CFRP structure and Dyneema skin were too great, it was necessary to refine the design of the skin to ensure that the structure and skin come together properly during the on-site fabrication and assembly of the kites.

To allow for the physical production of the fabric skin, the final relaxed form was again broken down into the four relaxed panels, each of which would then be panelized through MPanel into four smaller unique segments (i.e. P1_A, P1_B, P1_C, P1_D). This panelization allowed for the construction of the complex doubly curved surfaces from flat sheets of material such as the UHMWPE fabric [Figure 5]. As each of the four panels were longer than wide, the sub- 


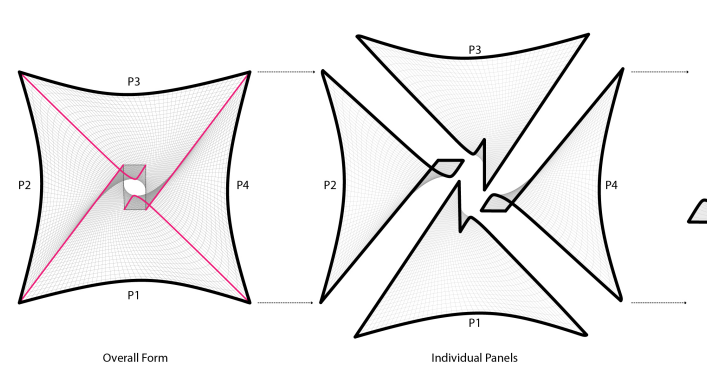

Individual Panels

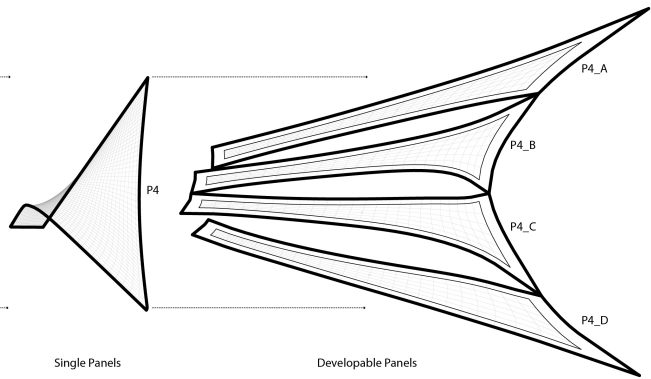

The plotted roll of nested sheets was then shipped to the site in Monteverde where each of the panels was manually cut out with an Xacto blade and assembled just as they were previously unrolled digitally utilizing the labeling and edge numbering. As the numbered edges were aligned, the form naturally took the double curved shape. The fabric panels were then welded through the application of DFC double sided fabric welding tape to both of the inner sides of the overlapping seams. In order to ensure proper attachment, pressure was applied to the seam during the welding process. This was repeated until all four panels were all connected together into one of the four sheets that comprised each kite skin. The sheet was not welded closed into its final form as it was first necessary to apply the microPCM.

\section{Microencapsulated Phase Change Material (microPCM)}

To reduce the temperature of the air and to increase the relative humidity of the air flowing through the prototypes, microPCM was applied to the interior surfaces of prototypes K-2 and K-3. Phase Change Material (PCM) was utilized because of its high thermal storage properties due to the storage of latent thermal energy as the material changes state from solid to liquid. Both prototypes utilized the same type of PCM, Rubitherm PX27, but had differing densities and patterns of the material. Rubitherm PX27 was chosen for use for several reasons. Firstly, this particular PCM was micro-encapsulated, which means
Figure 4

Prototype

panelization
Figure 5

Application of double sided tape for PCM embedding 
that the PCM was bound within a secondary support structure which contains the solid-liquid material during the phase change process. Without binding, the PCM would leak during its change of state from solid to liquid form. Secondly, the microPCM could easily be attached to the skin prototypes on site through the use of high strength double sided transfer tape. In addition, the melt temperature of the PCM was fairly constant between the temperatures of 24 to 27 degrees Celsius and the congealing area was between 27 and 24 degrees Celsius, which was believed to be a good working temperature for Monteverde's climate during the testing period in the summer months. The microPCM had a fairly high thermal storage capacity of around $95 \mathrm{~kJ} / \mathrm{kg}$. Finally, this specific PCM has stable phase change characteristics through repeat melt-solidification cycles.

Prior to welding the four sides of the fabric skin together, the micoPCM was attached to the interior surface of each skin using double-sided strips of transfer tape. Using the MPanel pattern as guidelines, the strips were placed in a variable pattern that increased in density towards the narrow throat of the air outlet. The pattern was intended to position more of the microPCM within the zone of low pressure air flow as observed in the CFD simulations. After the strips were in place, each panel was coated in an even layer of microPCM. Then, the four panels were welded together and stretched within their CFRP frames.

\section{ELECTRONICS}

In order to measure the change in air temperature and pressure, the two primary variables that the skin was designed to modify, the kites were equipped with microsensors at the air inlet and air outlet. Adafruit BME280 temperature, humidity, and pressure sensors were selected due to their size, availability, and cost. The sensors were connected to a portable microcontroller core, Adafruit Feather MO Adalogger, which included a built-in card reader, battery, USB connection, and switch to start and stop data recording remotely. The sensors were pro- grammed to transmit and record data every second. The sensors and microcontroller were attached to the kite prototypes within small pockets that allowed them to be removed and reinstalled during testing. Sensor 1, at air inlet, was located close to the tip of the kite frame in order to measure the properties of the air prior to modification by the form of the kite and the microPCM. Sensor 2 was positioned near the air outlet to determine the changes in the psychrometric properties of the air as it flowed through the kite. Both sensors were positioned on the inside of the fabric skin.

\section{ANALYSIS OF RESULTS}

The prototypes were tested over a period of three days in the Monteverde Cloud Forest Reserve in three locations selected due to their access to prevailing wind flows. One site was located along the main trail path, a second was positioned in a clearing near a forest opening, and the third was located along the ridge of the continental divide. The prototypes were brought to each location by foot, so all testing occurred along the major trails within the forest for ease of access.

The weather during testing shifted quickly and conditions were variable, with intermittent rain, clouds, sun, and changing wind speeds and direction. Both the sensors and microPCM were not adequately protected from moisture that collected on the inner surface the prototypes. Some of the sensors malfunctioned due to excess water accumulation at the connections. Furthermore, some of the microPCM did not remain in place due to water. Given these limitations, data was not collected from the smaller prototype, K-3. However, adequate and comparable data was collected from K-1 (without microPCM) and K-2 (with PCM).

Most of the testing of the kites was conducted with the sensors tethered to laptop computers in order to confirm that data was being collected and recorded. This also permitted the team to observe and record correlations in the performance of the kite with weather fluctuations in real time. Two people 


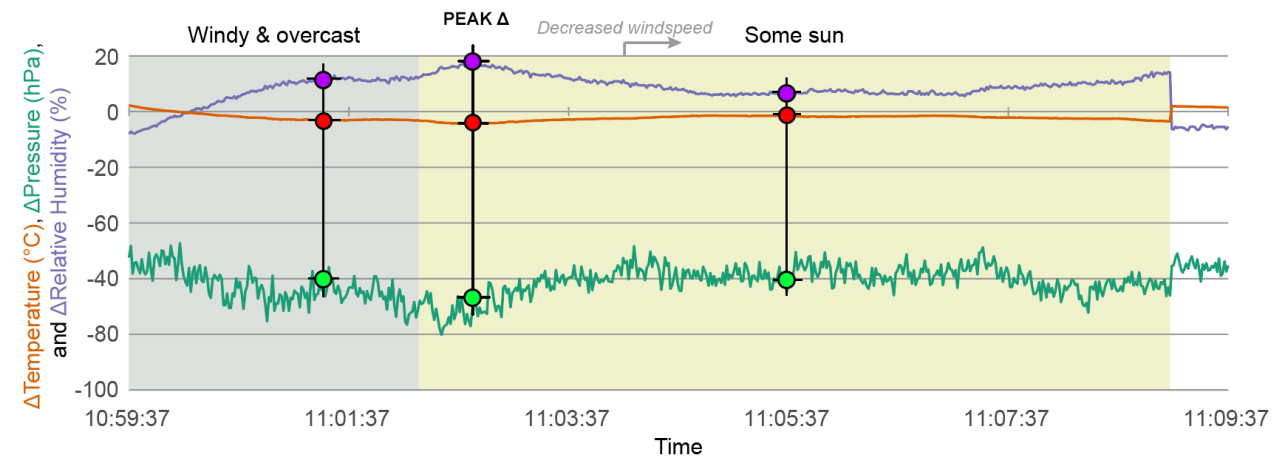

on the research team held the kite, one person monitored the climatic data on the computer screen, and two others recorded variations in weather conditions and recorded the timing and a description of the changes for further analysis. The comparative data supported that microPCM positively contributed to the performance of the kite with increased mean air temperature reductions observed in $\mathrm{K}-2\left(0.86^{\circ} \mathrm{C}\right)$ as compared to $\mathrm{K}-1\left(0.09^{\circ} \mathrm{C}\right)$, in similar weather conditions. [Ng, Wit, Hsieh, 2018] The testing of K-1 along the continental divide provided three notable correlations of the psychrometric air changes as follows [Figure 6]:

- In windy and overcast conditions, $12.18 \%$ increase in the relative humidity $(\mathrm{RH})$ of the air with a decrease in dry bulb temperature $(T)$ of $2.89^{\circ} \mathrm{C}$ [Figure 7]

- Peak changes occurred under sunny conditions with few visible clouds, $18.12 \%$ increase in $\mathrm{RH}$ with a decrease in $\mathrm{T}$ of $4.25^{\circ} \mathrm{C}$ [Figure 8]

- As the wind speed decreased, the performance of the kite decreased, with $6.32 \%$ increase in $\mathrm{RH}$ and decrease in $\mathrm{T}$ of $1.52^{\circ} \mathrm{C}$ [Figure 9]

Also notable in Figures 7 through 9 is the difference between target and actual performance, rang- ing from $2.06^{\circ} \mathrm{C}$ in windy and overcast conditions and the most humid air (condition 1); $3.35^{\circ} \mathrm{C}$ when peak changes in humidity (condition 2); and $2.78^{\circ} \mathrm{C}$ with decreased wind and the least humid air (condition 3 ).

\section{CONCLUSIONS}

Although this research only represents the results from the initial sets of scaled prototypes, it clearly shows potential in how form in conjunction with lightweight skins embedded with PCM can manipulate climatic conditions. By decreasing the internal air pressure and temperature, the prototypes were able to shift the properties of the air towards the $100 \%$ relative humidity curve at which water vapor would condense, but did not succeed. To further this research several aspects need to be reevaluated. First and foremost, the type, density and placement of the microPCM must be further investigated. The current prototypes utilized only a single melting temperature PCM, which was deemed too high to drop internal temperatures further. Future prototypes will utilize microPCM with a lower melt temperature, or several different microPCMs with variable melt temperatures placed strategically within the form, in order to increase the performative properties of the kite for this climate. In addition, full-scale kites would increase the surface area of the kite skin, thereby
Figure 6

K-1 correlations of psychrometric air changes 
Figure 7

Psychrometric charts depicting internal air changes: Figure 7: Overcast conditions; Figure 8: Sunny conditions; Figure 9: Decreased windspeed.
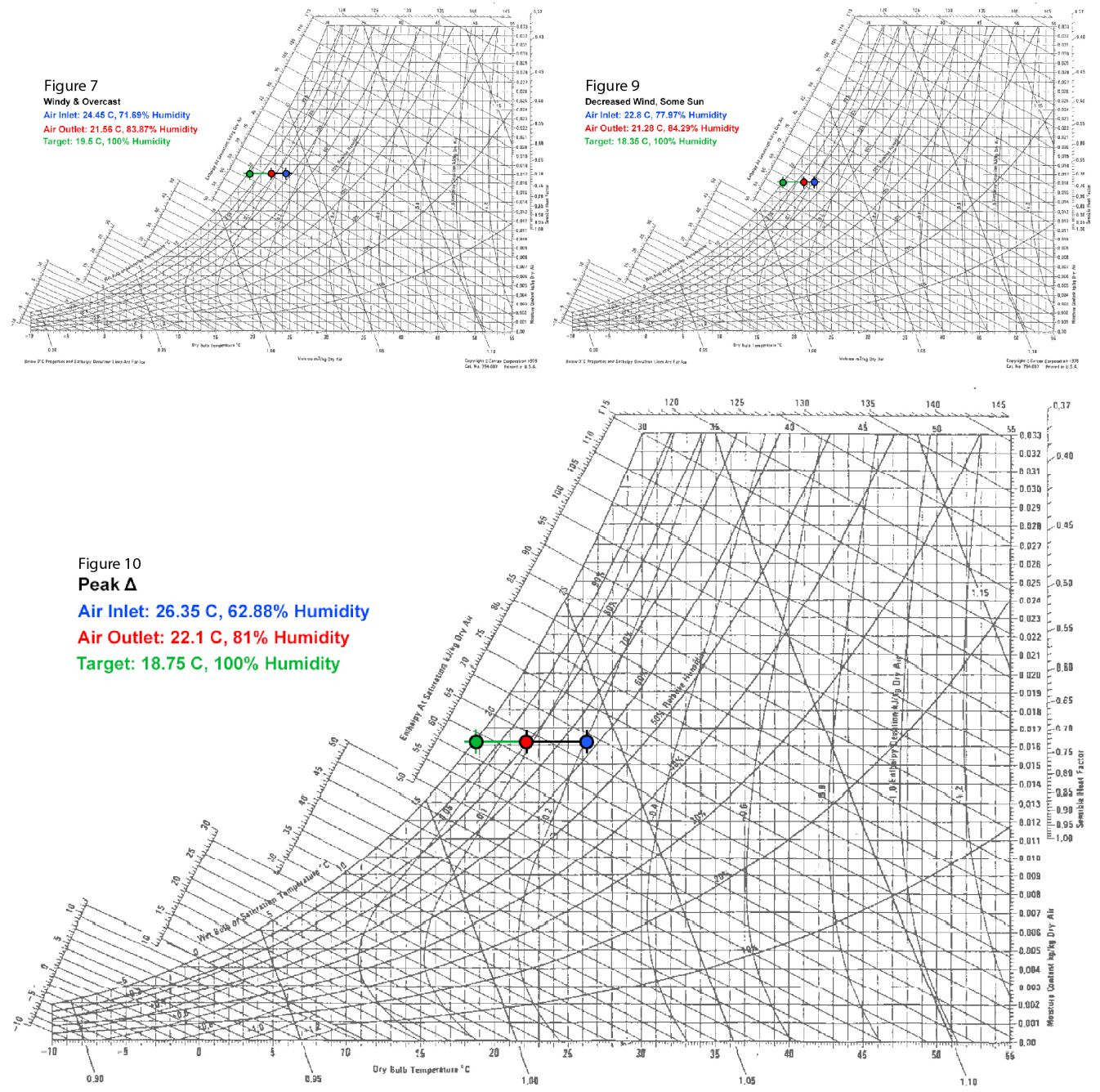
increasing the quantity of microPCM and the distance between inlet air and outlet air. Additionally, the form should be revised with a larger inlet and smaller outlet better allowing for greater decrease in air pressure which will also positively contribute to the condensation of water vapor. Furthermore, future prototypes will be designed to provide more water-resistant protection for the electronics and microPCM. These variables are expected to improve the performance of the kite in modifying the psychrometric properties of the air to meet the project goals.

Beyond the specific goals of cloudMAGNET, this research provides promising data in support of the potential to embed climatic mediating properties within lightweight fabrics. Such high-tech fabrics would provide more insulative properties within the facades of temporary or more permanent structures. These characteristics have previously only been capable through the use of heavier materials and assemblies. This would extend the use of fabric within architecture beyond its current applications, while also responding to the mandate for reduced energy usage and improved environmental performance.

\section{ACKNOWLEDGEMENTS}

This project was funded by a Temple University Presidential Humanities and Arts Research Program Award provided by Temple's Office of the Vice Provost for Research. The authors are grateful for the contributions and enthusiasm of Sneha Patel ('17 project leader; '14 Competition); Tonia Hsieh ('17 project leader); Justin Bernard ('17 assistant; '14 Competition); Rob Mertens and Hannah Candelaria ('17 participants); Sean Moss and Mary Stiger ('17 assistants, Philadelphia and Monteverde, Temple); Molly Berger, Natalie Duong, Sydney Farkas, Katie Fritz, Cori Keatts, Nikos Marks, Stephen Meyer, Jacob Schaffner, and Jenna Welch ('17 participants, James Madison); Cheng Zhang ('16 assistant, Temple University); Kerry Hohenstein ('15 assistant, Temple); Michael Farinella, Malgorzata Primavera, and Caroline Wineburg ('14 Competition).

\section{REFERENCES}

Faircloth, BF, Welch, RW, Sinke, Ys, Tamke, MT, Nicholas, PN, Ayres, PA, Eherenbard, EE and Ramsgaard Thomsen, MRT 2018 'Coupled Modeling and Monitoring of Phase Change Phenomena in Architectural Practice', Proceedings of the Symposium on Simulation for Architecture and Urban Design, pp. 81-88

Gramazio, FG and Kohler, MK (eds) 2014, Made by Robots: Challenging Architecture at a Larger Scale, Academy Press

Holmes, BH 2000, 'Case of the Dwindling Cloud Forest', International Wildlife, 30(4), pp. 20-27

$\mathrm{Ng}, \mathrm{RN}, \mathrm{WIT}, \mathrm{AJW}$ and Hsieh, TH 2018 'Cloud Magnet: the ethical imperative for environmental health and restoration', Proceedings of ARCC/EAAE 2018, Philadelphia, pp. 20-28

Rael, RR and San Fratello, VSF 2018, Printing Architecture: Innovative Recipes for 3D Printing, Princeton Architectural Press

Tibbets, ST 2016, Self-Assembly Lab: Experiments in Programming Matter, Routledge

Wit, AJW 2018 'cloudMAGNET, a CFRP Framework for Flexible Architectures', Proceedings of CAADRIA 2018, Beijing, pp. 49-58

[1] https://www.dsm.com/products/dyneema/en_US/t echnologies/dyneema-form-factors/fabric.html 